\title{
PECULIAR STARS OF LOW LUMINOSITY
}

\author{
J. LIEBERT \\ Steward Observatory \\ University of Arizona Tucson, AZ 85721 \\ AND \\ C.C. DAHN \\ U.S. Naval Observatory, Flagstaff Station \\ P.O. Box 1149 Flagstaff, AZ 86002-1149
}

\begin{abstract}
Precise trigonometric parallax measurements orders of magnitude more accurate than a milliarcsecond will contribute greatly to our understanding of peculiar, low luminosity stellar objects of several types. First, the volume of space out to which luminosities may be determined to the accuracy of the best, very-nearby stars will be increased greatly. For the relatively rare field Population II stars, this will lead to the first accurate empirical calibrations of the main sequence at the low mass end, for comparison with globular clusters of various metallicities. Parallaxes at $1 \mathrm{kpc}$ or farther will be adequate to help in the discovery or confirmation of the rare carbon dwarfs - main sequence stars with carbon-rich atmospheres. For cool white dwarfs, luminosities accurate to a few per cent or better will identify unresolved binaries, and objects of unusually high and low mass. For our most numerous solar neighbors, the $\mathrm{M}$ dwarfs and especially those near the stellar mass limit, accurate luminosities can help in the determinations of the chemical composition and age distributions.
\end{abstract}

\section{Introduction}

Trigonometric parallaxes approaching or exceeding 1 mas in accuracy have been obtained for very faint stars - i.e., down nearly to the limit of the Palomar Sky Survey - in the last decade using CCD detectors. This achievement has had substantial impact on our studies of several classes of low luminosity stars, which necessarily will be faint in apparent magnitude. These 
objects include many that are near the bottom of (1) the Population I main sequence, or dwarf M stars, (2) the Population II sequence, or subdwarf M stars, and (3) the white dwarf sequence, or degenerate dwarf stars. These parallaxes have provided the most reliable means of determining luminosity functions (LFs) for these classes of stars, or at least for calibrating the photometric systems used for this purpose.

In Fig. 1 we show an observational H-R Diagram based on U.S. Naval Observatory trigonometric parallaxes, both published and unpublished. All data points are shown with formal $\pm 1-\sigma$ error bars. Of course, the intrinsically faintest stars generally have the larger parallaxes and the smaller uncertainties. Only a few of these stars, generally in the top half of the diagram, are bright enough for HIPPARCHOS measurements. The accuracies of current parallaxes are good enough to demonstrate a dispersion among the $\mathrm{K}-\mathrm{M}$ dwarfs of $>3 \mathrm{mag}$, although the faintest of these at a given color are the extreme subdwarfs of Population II. Almost invariably, the most subluminous stars exhibit high tangential velocities, and low metallicities from their spectra and colors. However, due largely to their warmer temperatures and smaller bolometric corrections near the stellar mass limit, the Population II main sequence appears to terminate at $\mathrm{M}_{\mathrm{V}} \sim+14$ or slightly fainter, compared to $+18-19$ for the faintest stars of Population I. If the dispersion of the observed main sequence of nearby stars in $M_{v}$ were due primarily to the spread in metallicity, we would expect that below $M_{v}$ of +14 , the observed dispersion would grow smaller. Indeed, this is the appearance of the lower main sequence in Fig. 1. Of course, some remaining dispersion due to unresolved binaries and any pre-main sequence objects is also expected. We will comment later on the white dwarf sequence on the left side of the diagram.

In addition, parallaxes have led to the identification of unique and peculiar stars within these classes of low luminosity objects. One example of such an identification was the discovery nearly 20 years ago of the first dwarf carbon star (Dahn et al. 1977). Originally, this 13th magnitude object with a large proper motion was believed to be a low luminosity $M$ dwarf and, hence, was placed on the U.S. Naval Observatory parallax program. A fairly accurate trigonometric parallax permitted it to be placed in the $\mathrm{H}-\mathrm{R}$ diagram, but it had a peculiar location in $\mathrm{M}_{\mathrm{v}}$ vs. $\mathrm{B}-\mathrm{V}$ and $\mathrm{V}-\mathrm{I}$ colors compared to other $\mathrm{M}$ dwarfs or subdwarfs. Subsequent spectrophotometry obtained at Lick Observatory revealed a spectrum similar to the R-type giant stars, dominated by $\mathrm{C}_{2}$ Swan bands. Clearly the atmospheric composition of this star called G 77-61 is decisively different than observed in ordinary Population I dwarf stars! For a very long time G 77-61 remained the only known dwarf carbon star, and it was discovered because of the trigonometric parallax determintion. 


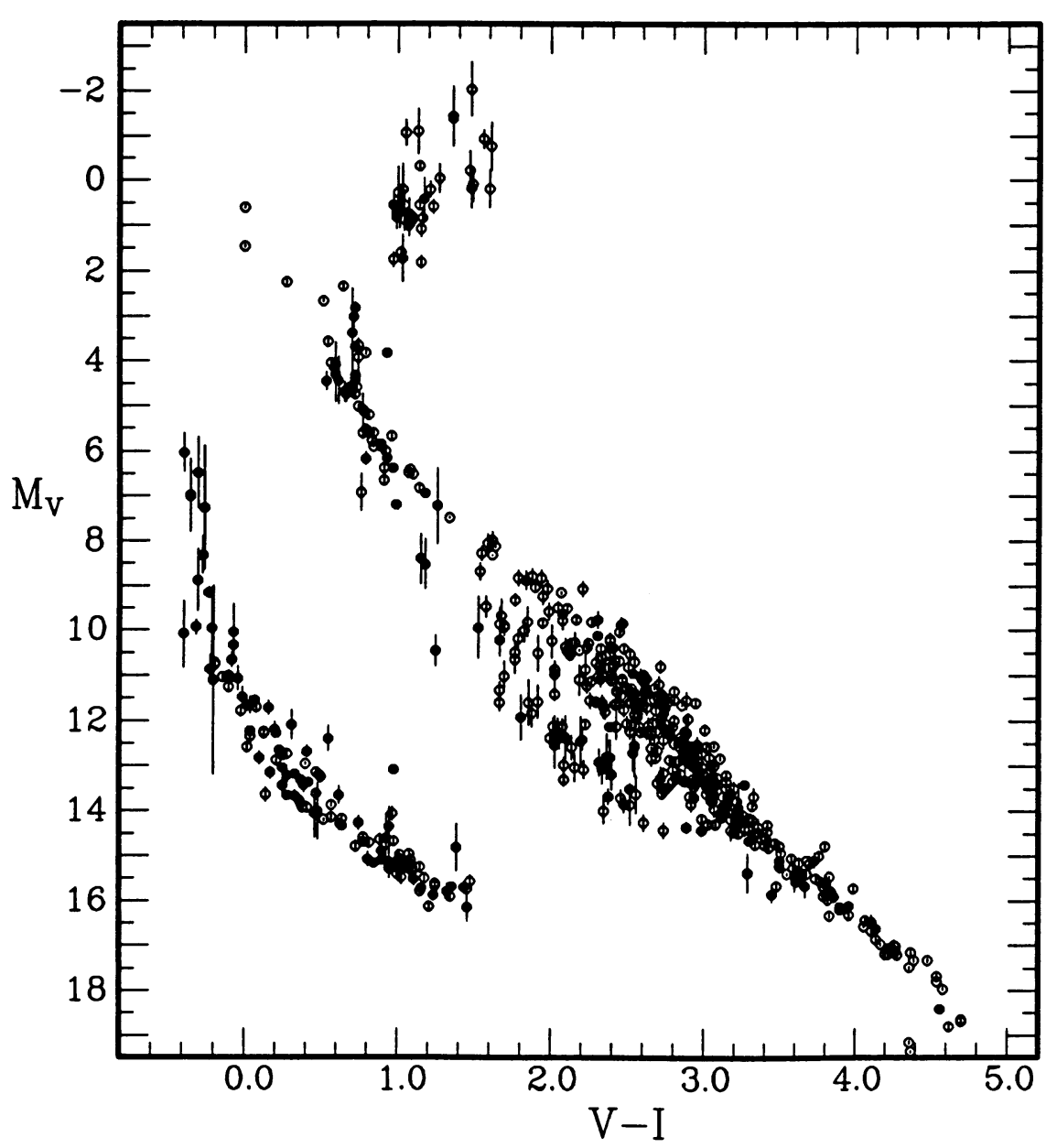

Figure 1. The $\mathrm{M}_{\mathrm{v}}$ vs $\mathrm{V}-\mathrm{I}$ diagram for a selection of 680 stars with USNO trigonometric parallaxes either published (open circles) or unpublished but nearly completed (filled circles).

When the accuracy of trigonometric parallaxes reaches an order of magnitude or two better than 1 mas, much more subtle peculiarities can be found in these classes of objects. Moreover, as we shall outline, global properties of the LFs and other parameters may be obtainable for large enough numbers of stars to be interesting. 


\section{Population II Stars in the Solar Neighborhood}

The Population II main sequence representatives in the Galactic disk near the Sun are of great potential importance for calibrating the main sequences in globular clusters. However, the space density of Population II stars is only of the order of $1 / 500$ that of their Population I counterparts, so one must search larger volumes of space to find rare objects. The achievement of submas parallaxes would mean a valuable combination of increased accuracy and a larger sample for this class of star. In the past the F-G subdwarfs have been used primarily for this purpose, because less accurate or no atmospheric abundance determinations have been available for $\mathrm{K}-\mathrm{M}$ subdwarfs; of course such abundances are necessary for comparison with the clusters, since the location of the main sequence is a function of metallicity.

Abundance estimates for $\mathrm{M}$ subdwarfs will be available soon, due largely to advances in model atmospheres analyses led by F. Allard, P. Hauschildt, K. Ruan, and their dissertation advisors R. Wehrse and M. Bessell. In a few years time, estimates of $[\mathrm{Fe} / \mathrm{H}]$ and specific heavy elements may rival the determinations for globular clusters, and the stars will then be useful calibrators if their distances are known.

\section{Main Sequence Carbon Stars}

We have already chronicled in the Introduction some of the history of work on G 77-61, perhaps the ultimate type of rare dwarf star. It has halo-like space motions and apparently a very low abundance of heavy elements (Gass, Liebert and Wehrse 1988), though incompleteness in the opacity sets appropriate for modeling such an unusual dwarf star may result in line abundances being underestimated. At $\mathrm{M}_{\mathrm{bol}} \sim+9$, it has the luminosity of an early $\mathrm{M}$ or late $\mathrm{K}$ dwarf, a bit more than $10^{-2} L_{\odot}$, and $\mathrm{T}_{\text {eff }}$ near $4,000 \mathrm{~K}$.

Its existence posed a problem for stellar evolution: Population II stars consistently show higher ratios of oxygen to carbon than stars of higher metallicity. Thus it is very unlikely that such a star formed out of material with $\mathrm{C} / \mathrm{O}>1$. The normal explanation for luminous carbon stars is that these are asymptotic giant branch (AGB) objects with carbon-oxygen cores whose contents gets mixed to the surface. However, a binary star interaction is the probable solution, as it so often is for stars with peculiar abundances: G 77-61 turns out to be a 243-day spectroscopic binary, though its likely white dwarf companion was too cool for detection with the International Ultraviolet Explorer Observatory (Dearborn et al. 1986).

In the last few years, new discoveries have increased the number of dwarf carbon stars to about ten (cf. Green, Margon and MacConnell 1991; Warren et al. 1993). Their coarse spectra appear to be remarkably similar in $T_{\text {eff }}$ to the prototype $\mathrm{G} 77-61$, yet the binary hypothesis should accom- 
modate a substantial distribution in the masses and other properties of the companions. Most, though apparently not all, of the new discoveries show kinematics of the halo population but none have abundance analyses. Two of the most recent discoveries are known to be binary, because a DA white dwarf spectrum is composite with the carbon dwarf spectrum.

Finally, the dwarf carbon stars complicate the job of those attempting to identify distant giant carbon stars in the halo, using their kinematics to study the mass distribution and structure of the extended halo. Hence, because of the evolutionary questions and galactic structure complications they pose, it is important both to find additional examples and to be able to recognize quickly this relatively rare class of star.

Sub-mas trigonometric parallaxes will permit the identification of dwarf carbon stars literally kiloparsecs away in the Galactic halo, since merely measuring a significant parallax will distinguish these from distant giants. For the modest sample now in hand, out to distances of at least $1 \mathrm{kpc}$, submas parallaxes would ensure accurate measurements of the luminosities, to see how uniform this distribution is.

\section{Cool and Peculiar White Dwarfs}

A large increase in the number and accuracy of parallaxes for, in particular, cool white dwarfs will make possible conclusions about the mass function as well as LF of these relics of the Galaxy's early star formation history. For hotter stars of spectral type DA, simultaneous fitting of accurate CCD Balmer line profiles has yielded values of $\mathrm{T}_{\text {eff }}$ and $\log g$ of unprecedented accuracy, and a very accurate relative mass distribution (Bergeron, Saffer and Liebert 1992). These hot DA stars represent the degenerates which have formed within the last Gyr or so.

For the cooler stars, such an analysis is impossible. The hydrogen lines are weak or absent, and the physics is far less accurate. Nevertheless, a spread in the mass distribution causes a dispersion in $M_{v}$ (i.e., radius) at a given color (i.e., temperature). In Fig. 1 there is some indication of the expected dispersion, but there are not many white dwarfs with welldetermined parallaxes at a given color.

Should accurate absolute visual magnitudes become available for many dozens of cool white dwarfs, however, one can plot the distribution of luminosities at a given color. Since color should correlate with temperature (even if precise values are unknown), the distribution of luminosities scales closely with the radius and can therefore map the mass distribution of white dwarfs as a function of cooling age. The sample would have to be at least several times larger in order to accomplish this goal, which means reaching out to larger distances with smaller parallax errors. 
White dwarfs with peculiar properties may also stand out in such diagrams based on sub-mas parallaxes. Actually, two of the cool white dwarfs in Fig. 1 that appear overluminous are the unresolved binaries L 870-2 and G $107-70$, at $\mathrm{V}-\mathrm{I}$ values near +0.6 and +1.0 , respectively. Here the measured luminosity comes from two similar stars. A second example might be strongly magnetic white dwarfs, which can have highly unusual energy distributions (Liebert 1988).

\section{Disk Dwarfs and Possible Substellar Counterparts}

We conclude with the largest and most important class of low luminosity objects, the low-mass main-sequence stars of Population I. Earlier, we discussed the appearance in Fig. 1 of a decreasing dispersion in $M_{v}$ fainter than +14 as the metal-poor contributors begin to disappear. There is predicted to be an opposite effect at a given metal abundance as one approaches the hydrogen-burning mass limit: the pre-main sequence time scale grows longer, approaching $1 \mathrm{Gyr}$. Then, below about $0.08 M_{\odot}$, there are objects of "transition mass," which succeed in sustaining part of their luminosity by a limited hydrogen-burning phase, before eventually developing degenerate interiors which quenches the burning. They then cool as brown dwarfs, but not before more than 1 Gyr has elapsed, during which they persist as objects of comparable luminosity to very low mass stars. Another way of stating the problem is to say that the dispersion in luminosity as a function of age increases greatly as the mass approaches and passes below the nuclear mass limit. If one is ever to sort out the stellar, the substellar and transition mass objects of low luminosity, accurate trigonometric parallaxes are indispensible.

\section{References}

Bergeron, P., Saffer, R.A., and Liebert, J. 1992, ApJ, 394, 228

Dahn, C.C., Liebert, J., Kron, R.G., Spinrad, H., and Hintzen, P.M. 1977, ApJ, 216, 757

Dearborn, D.S.P., Liebert, J., Aaronson, M., Dahn, C.C., Harrington, R., Mould, J., and Greenstein, J.L. 1986, ApJ, 300, 314

Gass, H., Liebert, J., and Wehrse, R. 1988, A\&A, 189, 194

Green, P.J., Margon, B., and MacConnell, D.J. 1991, ApJ, 380, L31

Liebert, J. 1988, PASP, 100, 1302

Warren, S.J., Irwin, M.J., Evans, D.W., Liebert, J., Osmer, P.S.,and Hewett, P.C. 1993, MNRAS, 261, 185 